\title{
Unusual Cause of Stridor with Failure to Thrive
}

\section{Jaya Madhava Reddy Chilukala ${ }^{1} \cdot$ Rashmi Kullu $^{1} \cdot$ B. Vishnu Bhat ${ }^{1} \cdot$ Satheesh Ponnarmeni $^{1}$}

Received: 9 July 2019 / Accepted: 5 August 2019 / Published online: 28 August 2019

(C) Dr. K C Chaudhuri Foundation 2019

To the Editor: A 27-d-old male neonate was brought with complaints of noisy breathing since $15 \mathrm{~d}$ of life and poor weight gain. He was born vaginally to 3rd gravid mother and had two normal elder siblings. The baby was normal at birth and weighed $3 \mathrm{~kg}$. In addition to breastfeeds, he was on top feeding because of poor sucking. On examination, he had inspiratory stridor and weighed $2.8 \mathrm{~kg}$. A provisional diagnosis of laryngomalacia with acute malnutrition was made. His hemoglobin, blood cell counts, serum electrolytes and renal function were normal. Urine examination revealed a few pus cells and the culture grew Escherichia coli, sensitive to Amikacin and Nitrofuradantin. Chest X-ray showed aspiration pneumonitis. The Otorhinolaryngologist suggested congenital laryngomalacia as the cause for stridor. The baby was treated with Amikacin for urinary tract infection and given tube feeding because of poor intake. The baby started gaining weight with expressed breast milk fed with orogastric tube. Brain MRI, done $15 \mathrm{~d}$ after the baby was not able to take oral feeds was normal.

In view of minimal stridor and inability to take full oral feed and inadequate weight gain, the baby was referred again to the Otorhinolaryngologist. Laryngoscopy revealed vallecular cyst obstructing the airway. Excision and marsupialisation of the cyst was done under general anesthesia and C-MAC guidance [1]. Post marsupialisation of the cyst, there was no inspiratory stridor and the baby could be fed well orally after 2 d. He was discharged on 60th $d$ on full breastfeeds with a weight of $3.72 \mathrm{~kg}$. The baby weighed $4.3 \mathrm{~kg}, 2 \mathrm{wk}$ later without any stridor.

Vallecular cyst is a rare life-threatening but treatable cause of stridor in neonates and infants. It arises beneath the mucosa of

B. Vishnu Bhat

drvishnubhat@yahoo.com

1 Department of Pediatrics, Pondicherry Institute of Medical Sciences, Kalapet, Pondicherry 605014, India the vallecula, either on the base of tongue or the lingual surface of the epiglottis. Because of inspiratory stridor and feeding difficulties, they can be misdiagnosed as laryngomalacia [2]. Most of these cysts are large enough and can be diagnosed using flexible laryngoscopy. We report this case because of its unusual presentation. In addition to larynogomalacia, the clinicians should be aware of other causes of inspiratory stridor and feeding difficulty $[3,4]$. Early diagnosis and specific treatment can improve the outcome.

\section{Compliance with Ethical Standards}

Conflict of Interest None.

\section{References}

1. Leibowitz JM, Smith LP, Cohen MA, Dunham BP, Guttenberg M, Elden LM. Diagnosis and treatment of pediatric vallecular cysts and pseudocysts. Int J Pediatr Otorhinolaryngol. 2011;75:899-904.

2. Parelkar SV, Patel JL, Sanghvi BV, et al. An unusual presentation of vallecular cyst with near fatal respiratory distress and management using conventional laparoscopic instruments. J Surg Tech Case Rep. 2012;4:118-20.

3. Yao TC, Chiu CY, Wu KC, Wu LJ, Huang JL. Failure to thrive caused by the coexistence of vallecular cyst, laryngomalacia and gastroesophageal reflux in an infant. Int J Pediatr Otorhinolaryngol. 2004;68:1459-64.

4. Ahrens B, Lammert I, Schmitt M, Wahn U, Paul K, Niggemann B. Life-threatening vallecular cyst in a 3-mo-old infant: case report and literature review. Clin Pediatr (Phila). 2004;43:287-90.

Publisher's Note Springer Nature remains neutral with regard to jurisdictional claims in published maps and institutional affiliations. 